\title{
The set of points at which a polynomial map is not proper
}

\author{
by ZBIGNIEW JELONEK (Kraków)
}

\begin{abstract}
We describe the set of points over which a dominant polynomial map $f=\left(f_{1}, \ldots, f_{n}\right): \mathbb{C}^{n} \rightarrow \mathbb{C}^{n}$ is not a local analytic covering. We show that this set is either empty or it is a uniruled hypersurface of degree bounded by $\left(\prod_{i=1}^{n} \operatorname{deg} f_{i}-\right.$ $\mu(f)) / \min _{i=1, \ldots, n} \operatorname{deg} f_{i}$.
\end{abstract}

1. Introduction. Let $f: \mathbb{C}^{n} \rightarrow \mathbb{C}^{n}$ be a dominant polynomial map. We say that $f$ is not proper at a point $y$ if there is no neighborhood $U$ of $y$ such that $f^{-1}(\operatorname{cl}(U))$ is compact, equivalently: if either $\operatorname{dim} f^{-1}(y)>0$ or $f^{-1}(y)=\left\{x_{1}, \ldots, x_{r}\right\}$ is a finite set but $\sum_{i=1}^{r} \mu_{x_{i}}(f)<\mu(f)$, where $\mu$ denotes multiplicity. The set of points at which $f$ is not proper indicates how far $f$ is from a finite map and therefore this set is important in the study of polynomial mappings (for example in the Jacobian Conjecture). Our aim is to describe this set precisely.

The main result is the following theorem:

TheOrem 15. Let $f=\left(f_{1}, \ldots, f_{n}\right): \mathbb{C}^{n} \rightarrow \mathbb{C}^{n}$ be a dominant polynomial map. Then the set $S$ of points at which $f$ is not proper is either empty or it is a uniruled hypersurface of degree not greater than

$$
\frac{\prod_{i=1}^{n} \operatorname{deg} f_{i}-\mu(f)}{\min _{i=1, \ldots, n} \operatorname{deg} f_{i}} .
$$

Moreover, $\operatorname{deg} f^{-1}(S) \leq \prod_{i=1}^{n} \operatorname{deg} f_{i}-\mu(f)$.

We also show how to find effectively an equation of the hypersurface $S$. In particular, we are able to check effectively whether a given polynomial mapping is proper.

The results of this paper were announced in [3].

1991 Mathematics Subject Classification: 14E05, 14E22.

Key words and phrases: polynomial mappings, proper mappings, dominant mappings, analytic covering. 
Acknowledgements. This paper was written during the author's stay at the Centre de Recerca Matematica of The Institut d'Estudis Catalans. I wish to express my gratitude to Prof. Manuel Castellet for his invitation and hospitality. I am also pleased to thank Prof. Arkadiusz Płoski for helpful remarks.

2. Terminology. Our notation and conventions are the same as in [5] and [9]. If $f$ is a dominant polynomial map and $\{x\}$ is an isolated component of the fiber $f^{-1}(f(x))$ we denote by $\mu_{x}(f)$ the multiplicity of $f$ at $x$ and by $\mu(f)$ the number of points in a generic fiber of $f$ (see, e.g., [5]).

Let $\mathbb{C}\left[z_{1}, \ldots, z_{n}\right]$ be a polynomial ring and $a_{1}, \ldots, a_{n}$ be positive integers. The weight of the monomial $a x_{1}^{k_{1}} \cdot \ldots \cdot x_{n}^{k_{n}}(a \neq 0)$ is, by definition, the number $\sum_{i=1}^{n} k_{i} a_{i}$. The weight of a polynomial $P$ is the maximal weight of its monomials (or $-\infty$ if $P=0$ ). It will be denoted by $v(P)$ and called the weight of $P$ determined by the conditions $v(x)=a_{i}$.

Let $X$ be an algebraic variety of dimension $n \geq 1$. X is said to be uniruled if there exists an algebraic variety $W$ of dimension $n-1$ and a dominant rational map $F: W \times \mathbb{P}^{1}(\mathbb{C})-\longrightarrow X$. Generally, if $X$ is an algebraic set of pure dimension $n \geq 1, X$ is said to be uniruled if every component of $X$ is uniruled. Equivalently, an algebraic set $X$ is uniruled if it has pure dimension $\geq 1$ and for a generic point in $X$ there is a rational curve in $X$ through this point.

3. Points at which a polynomial map is not proper. We begin with some basic and well-known facts about proper maps.

Definition 1. Let $X, Y$ be topological spaces. A continuous map $f$ : $X \rightarrow Y$ is said to be proper if for every compact set $K \subset Y$ the set $f^{-1}(K)$ is compact.

We have the following nice characterization of proper maps:

Proposition 2. Let $f: X \rightarrow Y$ be a continuous map of locally compact spaces. Then $f$ is proper if and only if it is closed and for every $y \in Y$ the set $f^{-1}(y)$ is compact.

If $X, Y$ are affine varieties there exists a well-known purely algebraic condition for a polynomial map $f: X \rightarrow Y$ to be proper.

Proposition 3. Let $X, Y$ be affine varieties and let $f: X \rightarrow Y$ be a polynomial map. Then $f$ is proper if and only if the map $f_{*}: \mathbb{C}[Y] \ni h \rightarrow$ $h \circ f \in \mathbb{C}[X]$ is finite, i.e., $f_{*} \mathbb{C}[Y] \subset \mathbb{C}[X]$ is an integral extension of rings.

Definition 4. Let $f: X \rightarrow Y$ be a continuous map. We say that $f$ is proper at $y \in Y$ if there exists an open neighborhood $U$ of $y$ such that $\operatorname{res}_{f^{-1}(U)} f: f^{-1}(U) \rightarrow U$ is a proper map. 
Remark 5. Assume that $X, Y$ are locally compact spaces. Then $f$ is proper if and only if it is proper at every $y \in Y$.

In the case of a polynomial map it is easy to show the following:

Proposition 6. Let $f: \mathbb{C}^{n} \rightarrow \mathbb{C}^{n}$ be a dominant polynomial map. Then $f$ is proper at $y \in \mathbb{C}^{n}$ if and only if $f^{-1}(y)=\left\{x_{1}, \ldots, x_{r}\right\}$ is a finite set and $\sum_{i=1}^{r} \mu_{x_{i}}(f)=\mu(f)$.

Now our main purpose is to give a description of the set of points at which a dominant polynomial map $f: \mathbb{C}^{n} \rightarrow \mathbb{C}^{n}$ is not proper. We start with the following fact:

Proposition 7. Let $f: \mathbb{C}^{n} \rightarrow \mathbb{C}^{n}$ be a dominant polynomial map and let $\mathbb{C}\left(f_{1}, \ldots, f_{n}\right) \subset \mathbb{C}\left(x_{1}, \ldots, x_{n}\right)$ be the induced field extension. Let

$$
\sum_{k=0}^{n_{i}} a_{k}^{i}(f) x_{i}^{n_{i}-k}=0,
$$

where the $a_{k}^{i}$ are polynomials, be an irreducible equation of $x_{i}$ over $\mathbb{C}\left[f_{1}, \ldots\right.$ $\left.\ldots, f_{n}\right]$. Let $S=\bigcup_{i=1}^{n}\left\{y \in \mathbb{C}^{n}: a_{0}^{i}(y)=0\right\}$. Then $f$ is proper at a point $y$ if and only if $y \in \mathbb{C}^{n} \backslash S$.

Proof. $\Rightarrow$ It is enough to prove that $f: \mathbb{C}^{n} \backslash f^{-1}(S) \rightarrow \mathbb{C}^{n} \backslash S$ is proper. If $S$ is empty then $\mathbb{C}\left[f_{1}, \ldots, f_{n}\right] \subset \mathbb{C}\left[x_{1}, \ldots, x_{n}\right]$ is an integral extension and by Proposition $3, f$ is proper. Otherwise $S$, and hence $f^{-1}(S)$, is a hypersurface. Set $A=\prod_{i=1}^{n} a_{0}^{i}$. Then $A$ is a non-constant polynomial and $S=\left\{y \in \mathbb{C}^{n}\right.$ : $A(y)=0\}, f^{-1}(S)=\{x: A(f(x))=0\}$. Let $V=\mathbb{C}^{n} \backslash f^{-1}(S)$ and $W=\mathbb{C}^{n} \backslash$ $S$. Then $V, W$ are affine varieties and $\mathbb{C}[V]=\mathbb{C}\left[x_{1}, \ldots, x_{n}\right]\left[(A(f(x)))^{-1}\right]$, $\mathbb{C}[W]=\mathbb{C}\left[y_{1}, \ldots, y_{n}\right]\left[A^{-1}\right]$. Hence $f_{*} \mathbb{C}[W]=\mathbb{C}\left[f_{1}, \ldots, f_{n}\right]\left[(A(f(x)))^{-1}\right]$. Since the $a_{0}^{i}(f)$ are units in $f_{*} \mathbb{C}[W]$ we conclude that the $x_{i}$ are integral over $f_{*} \mathbb{C}[W]$. Of course $A(f)^{-1}$ is also integral, and we get the integral extension $f_{*} \mathbb{C}[W] \subset \mathbb{C}[V]$. By Proposition 3 the proof of this part is finished.

$\Leftarrow$ The following lemma is proved in [10].

LEMMA 8. Let $\Omega$ be an open set in $\mathbb{C}^{n}, a_{i}: \Omega \rightarrow \mathbb{C}, i=0,1, \ldots, s$, be continuous functions with $a_{0} \neq 0$, and let $\Gamma=\{(x, t) \in \Omega \times \mathbb{C}$ : $\left.\sum_{i=0}^{s} a_{i}(x) t^{s-i}=0\right\}$. Then the projection $\pi: \Gamma \ni(x, t) \rightarrow x \in \Omega$ is proper if and only if $a_{0}(x) \neq 0$ for every $x \in \Omega$.

Consider the map $F=\left(f, x_{i}\right): \mathbb{C}^{n} \rightarrow \mathbb{C}^{n} \times \mathbb{C}$. It is a dominant map from $\mathbb{C}^{n}$ to the hypersurface given by the equation

$$
\sum_{j=0}^{n_{i}} a_{j}^{i}(y) x_{i}^{n_{i}-j}=0 .
$$

In particular, the image of $\mathbb{C}^{n}$ under $F$ is dense in the hypersurface $\Gamma$ given by the above equation. Assume that $f$ is proper at a point $y \in \mathbb{C}^{n}$. Then 
there exists an open neighborhood $U$ of $y$ such that $\operatorname{res}_{f^{-1}(U)} f: f^{-1}(U) \rightarrow$ $U$ is a proper map. We have the following diagram (here $\pi$ is the natural projection and the primes denote restriction):

$$
\begin{aligned}
& \begin{aligned}
F^{\prime} & U \times \mathbb{C} \\
f^{-1}(U) \stackrel{\downarrow}{f^{\prime}} & \downarrow
\end{aligned}
\end{aligned}
$$

Note that $F^{\prime}\left(f^{-1}(U)\right)$ is contained in $\Gamma \cap(U \times \mathbb{C})$ and dense in it. Since $\pi \circ F^{\prime}=f^{\prime}$ is proper, so is $F^{\prime}$. In particular, $F^{\prime}$ is closed, and consequently $F\left(f^{-1}(U)\right)=\Gamma \cap(U \times \mathbb{C}):=\Gamma^{\prime}$. This means that $F^{\prime}$ maps $f^{-1}(U)$ onto $\Gamma^{\prime}$.

Since $F^{\prime}$ is a surjection, for every compact set $K \subset U$ we have $\left(\operatorname{res}_{\Gamma} \pi\right)^{-1}(K)=F^{\prime}\left(f^{\prime-1}(K)\right)$, hence $\operatorname{res}_{\Gamma} \pi$ is a proper map. By Lemma 8 we conclude that $a_{0}^{i} \neq 0$ in $\Omega$, in particular, $a_{0}^{i}(y) \neq 0$. This proves the second part of Proposition 7 .

Corollary 9. Let $f: \mathbb{C}^{n} \rightarrow \mathbb{C}^{n}$ be a dominant polynomial map. Then the set $S$ of points at which $f$ is not proper is either empty or is a hypersurface.

Remark 10. a) In the same way as Proposition 7 we can prove the following theorem:

If $f: X \rightarrow Y$ is a dominant polynomial map of smooth affine varieties of the same dimension then the set of points at which $f$ is not proper is either empty or it is a hypersurface.

b) We can effectively find an equation of the hypersurface $S$. Indeed, by Proposition 7 and its proof it is enough to find equations of $n$ hypersurfaces which have parameterizations $F_{i}=\left(f, x_{i}\right), i=1, \ldots, n$.

This can be done in the standard way. For example it is enough to compute a Gröbner basis of the ideals $I_{k}=\left(y_{1}-f_{1}, \ldots, y_{n}-f_{n}, y_{n+1}-x_{k}\right)$, $k=1, \ldots, n$, with respect to the separating order. In this way we obtain polynomials $g_{k}(y) \in I_{k} \cap \mathbb{C}[Y], k=1, \ldots, n$, which are the equations we were looking for. If $g_{k}=\sum_{j=0}^{n_{k}} a_{j}^{k}(y) y_{n+1}^{n_{k}-j}$ then $S=\left\{y \in \mathbb{C}^{n}: \prod_{i=1}^{n} a_{0}^{i}(y)=0\right\}$.

In particular, we are able to check effectively whether a given polynomial mapping is proper.

Proposition 11. Let $f: \mathbb{C}^{n} \rightarrow \mathbb{C}^{n}$ be a dominant polynomial map and let $\mathbb{C}\left(f_{1}, \ldots, f_{n}\right) \subset \mathbb{C}\left(x_{1}, \ldots, x_{n}\right)$ be the induced field extension. For a given polynomial $h \in \mathbb{C}\left[x_{1}, \ldots, x_{n}\right]$ let

$$
\sum_{j=0}^{n_{k}} a_{j}^{h}(f) h^{m-j}=0,
$$

where the $a_{j}^{h}$ are polynomials, be an irreducible equation of $h$ over $\mathbb{C}\left[f_{1}, \ldots\right.$ $\left.\ldots, f_{n}\right]$. Then the set $\left\{y \in \mathbb{C}^{n}: a_{0}^{h}(y)=0\right\}$ is contained in the set $S$ of 
points at which $f$ is not proper. Moreover, if $h_{1}, \ldots, h_{p}$ are generators of $\mathbb{C}\left[x_{1}, \ldots, x_{n}\right]$ then $S=\bigcup_{i=1}^{p}\left\{y \in \mathbb{C}^{n}: a_{0}^{h_{i}}(y)=0\right\}$.

Proof. The proof is the same as above.

Proposition 12. Let $f=\left(f_{1}, \ldots, f_{n}\right): \mathbb{C}^{n} \rightarrow \mathbb{C}^{n}$ be a dominant polynomial map. The degree of the hypersurface $S$ of points at which $f$ is not proper is not greater than

$$
\frac{\prod_{i=1}^{n} \operatorname{deg} f_{i}-\mu(f)}{\min _{i=1, \ldots, n} \operatorname{deg} f_{i}} .
$$

Moreover,

$$
\operatorname{deg} f^{-1}(S) \leq \prod_{i=1}^{n} \operatorname{deg} f_{i}-\mu(f) .
$$

Proof. First we will show

LEMMA 13. There exists a linear polynomial $\eta=\sum_{i=1}^{n} c_{i} x_{i}\left(c_{i} \in \mathbb{C}\right)$ such that $S=\left\{x: a_{0}^{\eta}(x)=0\right\}$ and $\eta$ is a primitive element of the field extension $\mathbb{C}\left(f_{1}, \ldots, f_{n}\right) \subset \mathbb{C}\left(x_{1}, \ldots, x_{n}\right)$.

Proof. For $t \in \mathbb{C} \backslash\{0\}$ put $\eta(t)=\sum_{i=1}^{n} t^{i-1} x_{i}$. The family of hypersurfaces $S_{t}=\left\{x: a_{0}^{\eta(t)}(x)=0\right\}, t \in \mathbb{C} \backslash\{0\}$, is finite $\left(S_{t} \subset S !\right)$, hence there exists an infinite set $T \subset \mathbb{C} \backslash\{0\}$ such that $S_{t}=S_{t^{\prime}}$ for $t, t^{\prime} \in T$. Moreover, the family of subfields $\mathbb{C}\left(f_{1}, \ldots, f_{n}\right)(\eta(t)) \subset \mathbb{C}\left(x_{1}, \ldots, x_{n}\right), t \in T$, is also finite. It follows easily that there exist $n$ different numbers $t_{1}, \ldots, t_{n}$ (even infinitely many) such that $S_{t_{i}}=S_{t_{j}}$ and $\mathbb{C}\left(f_{1}, \ldots, f_{n}\right)\left(\eta\left(t_{i}\right)\right)=\mathbb{C}\left(f_{1}, \ldots, f_{n}\right)\left(\eta\left(t_{j}\right)\right)$ for every $i, j=1, \ldots, n$. Since $\eta\left(t_{1}\right), \ldots, \eta\left(t_{n}\right)$ are generators of the ring $\mathbb{C}\left[x_{1}, \ldots, x_{n}\right]$ over $\mathbb{C}$, all $\eta\left(t_{1}\right), \ldots, \eta\left(t_{n}\right)$ are primitive elements of the field extension $\mathbb{C}\left(f_{1}, \ldots, f_{n}\right) \subset \mathbb{C}\left(x_{1}, \ldots, x_{n}\right)$ and by Proposition 11 we conclude that $S_{t_{i}}=S$ for $i=1, \ldots, n$.

Now we need an old classical result due to O. Perron (see [6], Theorems 57 and 62 , and in a stronger modern version in [7], Theorem 1.5).

Let $F_{1}, \ldots, F_{m} \in \mathbb{C}\left[Z_{1}, \ldots, Z_{n}\right]$ be algebraically dependent polynomials of positive degrees and let $v$ be the weight in $\mathbb{C}\left[W_{1}, \ldots, W_{m}\right]$ determined by the conditions $v\left(W_{i}\right)=\operatorname{deg} F_{i}$, for $i=1, \ldots, m$. Then there exists a non-zero polynomial $P=P\left(W_{1}, \ldots, W_{m}\right)$ such that $P\left(F_{1}(Z), \ldots, F_{m}(Z)\right)=0$ in $\mathbb{C}[Z]$ and $v(P) \leq \prod_{i=1}^{m} \operatorname{deg} F_{i}$.

To prove Proposition 12, let $\eta$ be as in Lemma 13 and let $H(x, t)=$ $\sum_{j=0}^{m} a_{j}^{\eta}(x) t^{m-j}=0$, where the $a_{j}^{\eta}$ are polynomials, be an irreducible equation of $\eta$ over $\mathbb{C}\left[f_{1}, \ldots, f_{n}\right]$ (i.e., $\left.H(f, \eta)=0\right)$. Since $\eta$ is primitive, $m=\mu(f)$. We have to estimate the degree of $a_{0}^{\eta}$. We use Perron's theorem, where $m=n+1, F_{i}=f_{i}$ for $i=1, \ldots, n$ and $F_{n+1}=\eta$. Since $H$ divides $P$, 
we have $v(H) \leq v(P)$, hence $v(H) \leq \prod_{i=1}^{n+1} \operatorname{deg} F_{i}=\prod_{i=1}^{n} \operatorname{deg} f_{i}$. Thus $v\left(a_{0}^{\eta}\right) \leq v(H)-\mu(f) \leq \prod_{i=1}^{n} \operatorname{deg} f_{i}-\mu(f)$. Since

$$
\operatorname{deg} P \cdot \min _{i=1, \ldots, n} \operatorname{deg} f_{i} \leq v(P)
$$

for every polynomial $P$, we get

$$
\operatorname{deg} a_{0}^{\eta} \leq \frac{\prod_{i=1}^{n} \operatorname{deg} f_{i}-\mu(f)}{\min _{i=1, \ldots, n} \operatorname{deg} f_{i}} .
$$

Since $\operatorname{deg} S \leq \operatorname{deg} a_{0}^{\eta}$, we get the first estimate. To get the second one it is enough to note that $f^{-1}(S)$ has equation $a_{0}^{\eta}\left(f_{1}, \ldots, f_{n}\right)=0$ and $\operatorname{deg} f^{-1}(S) \leq \operatorname{deg} a_{0}^{\eta}\left(f_{1}, \ldots, f_{n}\right) \leq v\left(a_{0}^{\eta}\right) \leq \prod_{i=1}^{n} \operatorname{deg} f_{i}-\mu(f)$.

Proposition 14. Let $f: \mathbb{C}^{n} \rightarrow \mathbb{C}^{n}$ be a polynomial map with graph $\Gamma=$ $\left\{(x, y) \in \mathbb{C}^{n} \times \mathbb{C}^{n}: y=f(x)\right\}$. Denote by $\Gamma^{\prime}$ the closure of $\Gamma$ in $\mathbb{P}^{n}(\mathbb{C}) \times \mathbb{C}^{n}$ and put $R=\Gamma^{\prime} \backslash \Gamma$. Let $\pi$ denote the natural projection $\mathbb{P}^{n}(\mathbb{C}) \times \mathbb{C}^{n} \rightarrow \mathbb{C}^{n}$. Then the set $S$ of points at which $f$ is not proper is exactly the set $\pi(R)$. In particular, if $S$ is not empty then it is a uniruled hypersurface.

P r o o f. It is easy to see that $f$ is not proper at $y$ if and only if there exists a sequence $x_{n}$ such that $\lim x_{n}=\infty$ and $\lim f\left(x_{n}\right)=y$. This is equivalent to $y \in \pi(R)$.

By [2] every projective compactification of $\mathbb{C}^{n}$ is uniruled at infinity. Since $\Gamma$ is isomorphic to $\mathbb{C}^{n}$, every $(n-1)$-dimensional component of $R=$ $\Gamma^{\prime} \backslash \Gamma$ is uniruled, and consequently the hypersurface $S=\pi(R)$ must be uniruled.

We summarize Propositions 12 and 14 in:

TheOREM 15. Let $f=\left(f_{1}, \ldots, f_{n}\right): \mathbb{C}^{n} \rightarrow \mathbb{C}^{n}$ be a dominant polynomial map. Then the set $S$ of points at which $f$ is not proper is either empty or it is a uniruled hypersurface of degree not greater than

$$
\frac{\prod_{i=1}^{n} \operatorname{deg} f_{i}-\mu(f)}{\min _{i=1, \ldots, n} \operatorname{deg} f_{i}} .
$$

Moreover, $\operatorname{deg} f^{-1}(S) \leq \prod_{i=1}^{n} \operatorname{deg} f_{i}-\mu(f)$.

Theorem 15 has many interesting corollaries:

Corollary 16. Let $f$ and $S$ be as above. Then $\operatorname{deg} S<(\operatorname{deg} f)^{n-1}$, where $\operatorname{deg} f=\max _{i=1, \ldots, n} \operatorname{deg} f_{i}$. In particular, if $n=2$ then $\operatorname{deg} S<\operatorname{deg} f$.

Corollary 17. Let $f: \mathbb{C}^{n} \rightarrow \mathbb{C}^{n}$ be a dominant polynomial map. Then

$$
\mu(f) \leq \prod_{i=1}^{n} \operatorname{deg} f_{i}-\operatorname{deg} S \cdot \min _{i=1, \ldots, n} \operatorname{deg} f_{i},
$$

where $S$ denotes the hypersurface of points at which the map $f$ is not proper. In particular, if $\mu(f)>\prod_{i=1}^{n} \operatorname{deg} f_{i}-\min _{i=1, \ldots, n} \operatorname{deg} f_{i}$, then $f$ is proper. 
Corollary 18. Let $f$ be as above. Assume that $\mathrm{cl}\left(\mathbb{C}^{n} \backslash f\left(\mathbb{C}^{n}\right)\right)$ contains a hypersurface $H$ of degree $d$. Then $H$ is uniruled and

$$
d \leq \frac{\prod_{i=1}^{n} \operatorname{deg} f_{i}-\mu(f)}{\min _{i=1, \ldots, n} \operatorname{deg} f_{i}} .
$$

Corollary 19. Let $f: \mathbb{C}^{n} \rightarrow \mathbb{C}^{n}$ be a polynomial birational map which is not an automorphism. Then $\operatorname{cl}\left(\mathbb{C}^{n} \backslash f\left(\mathbb{C}^{n}\right)\right)$ is a uniruled hypersurface of degree

$$
d \leq \frac{\prod_{i=1}^{n} \operatorname{deg} f_{i}-1}{\min _{i=1, \ldots, n} \operatorname{deg} f_{i}} .
$$

ExAmple 20. Consider $f: \mathbb{C}^{n} \ni\left(x_{1}, \ldots, x_{n}\right) \rightarrow\left(x_{1}, \ldots, x_{n-1}, \prod_{i=1}^{n} x_{i}\right)$ $\in \mathbb{C}^{n}$. In this case $S=\operatorname{cl}\left(\mathbb{C}^{n} \backslash f\left(\mathbb{C}^{n}\right)\right)$ is the union of hyperplanes $\bigcup_{i=1}^{n-1}\{x$ : $\left.x_{i}=0\right\}$. Thus both degree estimates in Theorem 15 are sharp.

In the sequel we need the following generalization of a result of [4] (compare with [1]):

Proposition 21. Let $X$ be an affine set and $\Gamma$ be a closed affine subset of $X$. Let $f: \Gamma \rightarrow \mathbb{C}^{n}$ be a proper polynomial map and assume $n \geq$ $\operatorname{dim} X$. Then there exists a proper polynomial map $F: X \rightarrow \mathbb{C}^{n}$ such that $\operatorname{res}_{\Gamma} F=f$.

Proof. Let $I(\Gamma)$ denote the ideal of $\Gamma$ in $\mathbb{C}[X]$. Let $g=\left(g_{1}, \ldots, g_{n}\right)$ be a polynomial extension of $f$ to $X$. Let $x_{1}, \ldots, x_{m}$ be polynomial functions generating $\mathbb{C}[X]$ over $\mathbb{C}$, i.e., $\mathbb{C}[X]=\mathbb{C}\left[x_{1}, \ldots, x_{m}\right]$. It follows from our assumptions that there exist polynomial functions $a_{j}^{i} \in \mathbb{C}\left[X_{1}, \ldots, X_{n}\right]$ such that $H_{i}=\sum_{j=0}^{n_{i}} a_{j}^{i}(g) x_{i}^{n_{i}-j}=0 \bmod I(\Gamma)$, and $a_{0}^{i}(g)=$ const $\neq 0$ for $i=1, \ldots, m$. Consider the map $H=\left(g_{1}, \ldots, g_{n}, H_{1}, \ldots, H_{m}\right): X \rightarrow \mathbb{C}^{n+m}$. By construction $H$ is proper, $H(\Gamma) \subset \mathbb{C}^{n} \times\{0\}$ and $\operatorname{dim} H(X)=\operatorname{dim} X \leq n$. The set $Y:=H(X)$ is a closed subset of $\mathbb{C}^{n+m}$. It is easy to see that there exists a proper linear projection $\pi: Y \rightarrow \mathbb{C}^{n} \times\{0\}$.

Indeed, we have the canonical inclusion $\mathbb{C}^{n+m} \subset \mathbb{P}^{n+m}(\mathbb{C})$ and the set $W$ of points at infinity of $Y$ has dimension $\leq n-1$. Hence we can find a linear subspace $L$ of the hyperplane at infinity of dimension $m-1$ which is disjoint from $W$. Let $\pi_{L}: \mathbb{P}^{n+m}(\mathbb{C}) \rightarrow \operatorname{cl}\left(\mathbb{C}^{n} \times\{0\}\right)$ be the projection determined by $L$. The restriction of $\pi_{L}$ to $Y$ is a proper map of $Y$ into $\mathbb{C}^{n} \times\{0\}$ and it is the projection $\pi$ we were looking for.

Now it is enough to take $F=p \circ H$ to obtain a proper extension of $f$ to the whole of $X$.

Remark 22. It is easy to see that Proposition 21 remains true if we replace "proper polynomial map" by "polynomial map with generically finite fibers". 
EXAMPLE 23. Let $S \subset \mathbb{C}^{2}$ be a "parametric" rational curve, i.e., $S$ is the image of $\mathbb{C}$ under a non-constant polynomial mapping. Then there exists a polynomial mapping $F: \mathbb{C}^{2} \rightarrow \mathbb{C}^{2}$ with finite fibers for which $S$ is exactly the set of points at which $F$ is not proper.

Indeed, let $V=\left\{(x, y, z) \in \mathbb{C}^{3}: z^{2}-x y-1=0\right\}$. Then $V$ is an affine variety and there exists a (non-proper) embedding $i: \mathbb{C}^{2} \ni(u, v) \rightarrow(u(2+$ $u v), v, 1+u v) \in V$. Note that $V \backslash i\left(\mathbb{C}^{2}\right)$ is the line $l=\{z=-1, y=0\} \cong \mathbb{C}$. If $\sigma: l \rightarrow S$ is a parameterization then by Proposition 21, $\sigma$ can be extended to a proper map $\Sigma: V \rightarrow \mathbb{C}^{2}$. Now it is enough to take $F=\Sigma \circ i$.

\section{References}

[1] Z. Jelonek, The extension of regular and rational embeddings, Math. Ann. 277 (1987), 113-120.

[2] -, Irreducible identity sets for polynomial automorphisms, Math. Z. 212 (1993), 601-617.

[3] - The set of points at which a polynomial map is not proper, preprint CRM no. 141, Bellaterra, March 1992.

[4] M. Kwieciński, Extending finite mappings to affine spaces, J. Pure Appl. Algebra 76 (1991), 151-154.

[5] D. Mumford, Algebraic Geometry I, Springer, Berlin, 1976.

[6] O. Perron, Algebra I (Die Grundlagen), Göschens Lehrbücherei, Berlin und Leipzig, 1932.

[7] A. Płoski, Algebraic dependence and polynomial automorphisms, Bull. Polish Acad. Sci. Math. 34 (1986), 653-659.

[8] -, On the growth of proper polynomial mappings, Ann. Polon. Math. 45 (1985), 297-309.

[9] I. R. Shafarevich, Basic Algebraic Geometry, Springer, 1974.

[10] P. Tworzewski and T. Winiarski, Analytic sets with proper projections, J. Reine Angew. Math. 337 (1982), 68-76.

INSTITUTE OF MATHEMATICS

JAGIELLONIAN UNIVERSITY

REYMONTA 4

30-059 KRAKÓw, POLAND 